\title{
Cahiers d'ethnomusicologie
}

Anciennement Cahiers de musiques traditionnelles

\section{Musiques à écouter, musiques à voir : la musique dans les musées de société}

Compte rendu des Journées d'Étude de la Société Française

d'Ethnomusicologie, Carry-le-Rouet, 24-26 mai 2002

Florence Gétreau et Laurent Aubert

\section{(2) OpenEdition}

Journals

Édition électronique

URL : http://journals.openedition.org/ethnomusicologie/836

ISSN : 2235-7688

Éditeur

ADEM - Ateliers d'ethnomusicologie

Édition imprimée

Date de publication : 1 novembre 2002

Pagination : 237-246

ISSN : 1662-372X

Référence électronique

Florence Gétreau et Laurent Aubert, « Musiques à écouter, musiques à voir : la musique dans les musées de société », Cahiers d'ethnomusicologie [En ligne], 15 | 2002, mis en ligne le 11 janvier 2012, consulté le 01 mai 2019. URL : http://journals.openedition.org/ethnomusicologie/836

Ce document a été généré automatiquement le 1 mai 2019.

Tous droits réservés 


\section{Musiques à écouter, musiques à voir : la musique dans les musées de société}

Compte rendu des Journées d'Étude de la Société Française

d'Ethnomusicologie, Carry-le-Rouet, 24-26 mai 2002

\section{Florence Gétreau et Laurent Aubert}

1 La richesse et la densité de ces Journées de la SFE nous a paru suffisante pour justifier la publication de ces deux comptes rendus, qui en proposent des regards complémentaires. Le programme détaillé des Journées figure sur le site de la Société Française d'Ethnomusicologie < http://www.ethnomusicologie.fr/>. Signalons par ailleurs qu'il est prévu que le dossier du prochain volume des Cahiers de musiques traditionnelles (16/2003) soit entièrement consacré au thème de la musique dans les musées (réd.).

\section{Synthèse et perspectives}

2 C'est l'actualité des musées de société, les grands bouleversements en cours ou programmés qui ont suggéré à la Société Française d'Ethnomusicologie l'idée de ces journées d'étude. Musée de l'Homme, Musée des Arts océaniens et africains, Musée du Quai Branly d'un côté, Musée national des Arts et Traditions populaires et futur Musée des civilisations de l'Europe et de la Méditerranée de l'autre posent en effet la question de l'intégration de la musique dans ces nouvelles institutions, tant au plan des collections, des expositions, des activités que de la recherche. Mais bien d'autres institutions en France contribuent à cette dynamique et à ce questionnement: le musée de la Musique, qui prépare des réajustements dans son parcours et ses activités, plusieurs musées régionaux dont certains, comme celui de Montluçon, seront très largement consacrés à la musique.

3 L'organisation des journées d'étude à Marseille avait donc valeur symbolique. Pascale Porret et Laurence Fayet ont offert une parfaite organisation dans ce lieu magnifique et particulièrement inspirant entre ciel et mer. Chacun aura noté l'équilibre des thématiques (permettant de rendre compte des expériences accumulées, des média 
disponibles, des publics concernés), la richesse des communications - dont certaines ont été écourtées en raison de la grande densité des sessions - et de la qualité des échanges, reflet de nos préoccupations dans la variété de nos métiers (ethnomusicologues, muséologues, musiciens, responsables d'associations, concepteurs de produits et systèmes techniques).

\section{Quelques constats}

Dans les réalisations anciennes ou plus récentes (notamment celles présentées dans «Réalisations récentes, expériences en cours » et dans «La matière sonore, sa présence dans le Musée »), que ce soit dans le cas d'expositions permanentes ou de manifestations temporaires, la musique n'est pas assez présente : que ce soit la possibilité d'écouter, que ce soit l'appréhension de la matière musicale, que ce soit les outils de perception ou d'analyse, ils font presque toujours défaut, même quand le sujet est foisonnant et les collections abondantes.

5 L'objet matériel est remis en cause. La place et la fonction des objets, c'est-à-dire principalement des collections d'instruments de musique, semblent poser question. Veuton exposer les collections d'instruments de musique ou la musique ? La dénomination du Comité international des musées et collections d'instruments de musique (CIMCIM) au sein de l'ICOM (Conseil international des musées) n'est-elle pas pour partie obsolète ? La voix et la danse sont trop souvent les parents pauvres des sections musicales des musées et expositions.

6 La fonction et les contraintes des collections de musées semblent devoir être clarifiées : répondre aux demandes du public, par une approche participative, expérimentale, voire utilitaire de l'instrument, implique probablement de concevoir, auprès des collections patrimoniales, des collections éducatives constituées de fac-simile d'instruments, qui seraient vouées aux actions culturelles et pédagogiques. Mais on ne peut confondre leurs vocations : beaucoup d'instruments conservés dans les collections de musées constituent aujourd'hui des « espèces en voie de disparition » qu'il faut bien considérer comme des documents et non plus comme des outils destinés à la pratique.

7 L'histoire des collections publiques montre que leur géographie n'a jamais été statique. La logique de la collectivité l'a toujours emporté sur les souhaits des multiples individus qui ont constitué les collections (princes, savants, hommes de terrain, amateurs). Les instruments rapportés par le père Amiot pour le Cabinet du Roy n'avaient-ils pas eu vocation à être intégrés au Musée d'ethnographie en 1878 ? Et si c'est le cas, pourquoi ne changeraient-ils pas de localisation et donc d'affectation une nouvelle fois? De ce point de vue, on ne peut pas condamner a priori le transfert des collections du Musée de l'Homme au futur Musée du Quai Branly. Tous nos musées sont le fruit de ces mouvements de collections (agrégations, désagrégations) liés aux opportunités et aux politiques patrimoniales. La redéfinition en cours des terrains d'action rend ce réseau d'échange, d'emprunts et de collaboration d'une particulière actualité.

8 Les options retenues pour le Musée du Quai Branly ont été largement débattues. Elles ne semblent pas recueillir l'adhésion de la communauté scientifique que nous formons :

- On a relevé les contradictions qu'incarne le «silo", cette réserve d'instruments transparente, à volumétrie cylindrique et strates empilées, placée au coeur du futur parcours muséographique - un tel paradoxe est sans doute le symptôme de la mise en question du rôle des collections, mais aussi de la difficulté à faire émerger des 
propositions originales. On ne peut que s'interroger sur l'adéquation d'un tel espace avec sa fonction : les performances techniques se ramènent à des compromis puisque priorité a été donnée à l'idée architecturale (cette réserve est un axe important de la composition architecturale). Aussi chacun se demande si luminosité, poussière, circulation, conditionnement ont été étudiés pour une protection et une accessibilité optimale des collections.

- La dynamique que constitue le chantier des collections, avec ses enjeux documentaires et son souci d'une conservation préventive professionnelle a été saluée car elle a fait trop longtemps défaut. Apparaît là aussi une opportunité à saisir: que l'élaboration des déontologies, des méthodes, des outils conceptuels (thesaurus par exemple), et donc des décisions se fasse dans un processus de large consultation, voire de concertation, puisque de nombreux musées musicaux ou à départements musicaux en cours de transformation sont face à des choix de même nature.

\section{De nombreuses propositions}

9 Rappelons le cadre théorique de réflexion proposé chacun dans leur domaine par Michel Colardelle et par Bernard Lortat-Jacob.

Pour le premier, le futur Musée de l'Europe et de la Méditerranée ne se définit pas par «le populaire ", mais bien comme "l'ensemble des traits élaborés dans une génétique d'emprunts et de rejets, voire de violence, fabricant des systèmes identitaires ». Il doit rendre compte du processus d'élaboration, des évolutions, de la construction des différences, des grands systèmes symboliques, et poser les grands questionnements de la société. S'appuyant sur le comparatisme et se présentant comme le résultat toujours provisoire d'une proposition, le musée est un lieu de connaissance, mais aussi d'appréciation et pourquoi pas de délectation. Ses expositions de référence souligneront autant les systèmes techniques que symboliques. Croisant le regard de l'historien, du sociologue, du linguiste, il demande à l'ethnologue de poser des questions plutôt que de les résoudre. Il place en revanche le public au coeur d'un processus de transmission, de mobilité, de contact.

Réaffirmant le principe d'un musée-laboratoire, Michel Colardelle insiste sur la nécessaire transdisciplinarité $\mathrm{du}$ projet, qui doit s'appuyer sur une recherche extrêmement forte, les chercheurs du laboratoire apportant leur capacité de synthèse (à mettre en résonance avec les préoccupations de diffusion des connaissances vers le public qui sont celles des muséologues).

12 A fortiori la musique doit-elle trouver un nouveau langage au sein d'un tel musée. Le langage des objets n'est pas suffisant. Il faut donc une révolution mentale pour que le musée se raconte aussi par les sons et les bruits. Il faut aussi que ses espaces soient d'une grande plasticité d'utilisation afin d'offrir une réelle mobilité d'activité.

Bernard Lortat-Jacob, entrant dans le vif du sujet, propose pour sa part tout d'abord une réflexion conceptuelle sur la musique, celle des ethnomusicologues. Il souligne la difficulté qu'il y a à représenter une représentation, à partager la musique puisque l'objet inclut le sujet. Comment rendre publique et donc collective une représentation intime et donc subjective ? L'inventaire des outils de connaissance, qu'ils soient médiatisés ou non (avec notamment enregistrement sonore, film, notation musicale, transcription électroacoustique, représentations graphiques, littérature), et la mise en évidence du rôle des passeurs (les musiciens, les ethnomusicologues) montrent que chacun pris 
séparément donne une définition trop réductrice du fait musical. C'est donc avec une palette de clefs d'écoute qu'il propose de constituer un cadre fort de propositions pour l'élaboration de thématiques à muséographier. A partir de la connaissance subjective d'un sujet, elles consisteraient à scénographier des lieux de prédilection, faire toucher la matière sonore (par un dispositif interactif), déboucher sur une approche documentaire (par un film qui constituerait une sorte d'aboutissement), chacun pouvant « raconter son histoire ".

propositions concrètes venant d'autres ethnomusicologues soulignent presque toutes la relation entre musique/culture/codes et invitent à donner une place équilibrée à ces composantes dans la partie publique du musée.

Les expériences multimedia d'analyse musicale (celles présentées respectivement par Marc Chemillier et Susan Fürniss), quoique considérées par leurs concepteurs comme destinées à des chercheurs, ne semblent pas si éloignées de celles qui ont été élaborées en vue d'un très large public pour le «Secteur Son » de la Cité des Sciences et de l'Industrie à La Villette. Grâce au sonogramme, Marc Chemillier nous fait par exemple pénétrer dans le processus subtil de production de la quintina, une cinquième note harmonique qui n'est produite à strictement parler par aucun des quatre chanteurs du choeur, mais qui en résulte selon un mécanisme acoustique jusque-là mystérieux. Or cette cinquième note est considérée par les chanteurs comme un symbole de pa présence de la Vierge lors du Chant de la Passion à Castelsardo, en Sardaigne. N'est-il pas alors hors de propos de penser que le public est rebuté par la découverte de phénomènes relativement complexes? Après ces essais faits il y a déjà quelques années on ne peut que souhaiter que de nouvelles et nombreuses suggestions soient mises en oeuvre.

Parmi les multiples expériences relatées, retenons les remarques générales et "pragmatiques » d'olivier Tourny, lequel insiste sur le caractère "provisoire » des concepts qui marquent la création d'un projet muséal. Il recommande d'avoir une approche adaptée selon que le projet est national et porte sur une culture nationale ou étrangère ou que le projet est local et porte sur une culture autochtone.

17 Pour Pais de Brito, le travail avec une équipe pluridisciplinaire est seule garante du résultat. Même au stade de la collecte, il y a nécessité à faire appel à des spécialistes et de travailler le son indépendamment de la source. Dans une exposition sur le fado montée il y a quelques années à Lisbonne, le défi était de taille puisqu'il n'y avait que très peu d'objets et d'instruments à montrer. Il fallut donc mettre en valeur le geste musical, l'émotion et le grain de la voix grâce à des moyens audiovisuels utilisés à bon escient.

Rosalia Martinez, recommandant de son côté de ne pas opposer la connaissance et le sensible, propose de montrer les équivalences entre contrastes de couleurs dans le costume et contrastes de sons dans les musiques de carnaval andines, contrastes qui ont, dans ces deux domaines, une valeur cosmologique en relation avec l'ordre et le désordre. Luc Martinez souligne quant à lui la nécessité que le contenu de l'exposition soit conçu et élaboré au maximum en amont de la réalisation technique de la mise en espace sonore, car cette dernière doit s'adapter au contenu, et non l'inverse.

Enfin, pour Philippe Fanise et Eric Montbel, le musée doit jouer un rôle social par la diversité des pratiques culturelles qu'il propose, par le réseau de communautés qu'il reflète. Espace de référence, il est un outil de réflexion et de rencontre par excellence. 


\section{Une méthode de travail dans la perspective du futur musée de Marseille}

21 A propos du chantier des collections qui va débuter en 2004, constatons que trois domaines concernent déjà la musique: les collections sonores du MNATP/CEF ont commencé à être numérisées (par transfert sur $\mathrm{CD}$ ) grâce au plan national de numérisation lancé par le Ministère de la Culture en 1999. Quelque 1000 heures sont déjà transférées; l'informatisation de ces collections sonores, mais aussi des fonds de partitions de chansons, est prévue à partir de 2005; le catalogue scientifique des instruments de musique est en cours.

Le son et la musique ont été pris en compte dès la rédaction du programme architectural remis aux équipes d'architectes concourrant en vue de la désignation d'un maître d'oeuvre. Son et musique inerveront les différentes activités du musée : que ce soit dans les expositions de référence, dans les lieux de rencontre qui les ponctueront, dans les espaces pédagogiques et documentaires, dans une approche à la fois sensible et constructive de savoirs grâce à la variété des media, des lieux, des formules.

La présence de la recherche en ethnomusicologie dans le futur établissement est une nécessité. Nous avons une responsabilité collective pour assurer une relève au musée. A tout le moins, un réseau rapproché d'expertise et de propositions est indispensable pour construire le projet de Marseille. Il sera complémentaire des campagnes de collecte et des enquêtes sociologiques sur les pratiques musicales contemporaines menées notamment par les sociologues de la maison.

24 Le séminaire d'anthropologie comparative organisé avec Bernard Lortat-Jacob dans le cadre de la convention associant l'IDEMEC (Maison méditerranéenne des Sciences de l'homme) et le MNATP/CEF, le 22 novembre 2002 sur le thème Musiques traditionnelles: représentations, espaces, enjeux constitue la nouvelle étape de ce dialogue.

Florence Gétreau

\section{Le paradoxe du musée vivant}

L'intégration de la musique dans un musée procède en soi d'un paradoxe, qu'on pourrait appeler le paradoxe du musée vivant. En effet, le musée n'est-il pas ce bel écrin où reposent des chefs-d'oeuvre inanimés, définitivement privés de toute utilité, sinon celle d'être livrés à la délectation des visiteurs ? Le musée donne à voir, à sentir, à apprendre et, éventuellement, à réfléchir. Or la musique n’a jamais été conçue pour être montrée, mais pour être vécue et partagée.

Comment, dès lors, traiter la musique et, plus largement, le son dans les musées? Que voulons-nous exposer de la musique? Comment la présenter et la faire comprendre? Quelle doit enfin être la place de la musique vivante dans un tel processus? Telles sont les interrogations initiales sur lesquelles se sont articulées ces trois journées de la Société Française d'Ethnomusicologie. Par la diversité des regards et des compétences qu'elles ont mis en jeu, par les confrontations même qu'elles ont suscitées, elles ont bien posé les bases d'une évaluation commune de la place de la musique dans les musées de société, tout en redéfinissant le rôle que ses spécialistes - ethnomusicologues, organologues, scénographes ou acousticiens - sont amenés à jouer dans sa détermination. 
27 Les réflexions développées à Carry-le-Rouet du 24 au 26 mai 2002 ont permis de dégager un certain nombre de thèmes récurrents, de rubriques sous lesquelles peuvent être regroupés les constats, les préoccupations et les propositions des participants.

\section{Où la musique est-elle montrée ?}

28 -Un musée consacré à La musique : les musiques qui nous occupent risquent de s'y trouver dans une position de parent pauvre vis-à-vis de la musique classique occidentale. L'exemple du Musée de la Musique de la Villette, présenté par Philippe Bruguière, illustre bien le type de contraintes auquel une telle juxtaposition soumet les «instruments du monde ». En effet, l'absence, dans les salles d'exposition, de toute iconographie, de tout appareil didactique, peut à la rigueur être revendiquée pour ce qui concerne les instruments de la musique «dominante ». Elle ne se justifie en revanche pas dans le cas d'objets qui, une fois sortis de leur contexte, ne livrent qu'une part infime de leurs secrets.

29 - Un musée d'art: l'art dans un musée étant en principe toujours l'art visuel, la présentation de la musique offre le risque d'y être réduite à une approche esthétisante, se résumant à l'exhibition de quelques « beaux » objets. Les propos de Madeleine Leclair et de Germain Viatte sur le projet du Musée du quai Branly à Paris, aussi séduisants fussentils, ne sont pas parvenus à dissiper les craintes sur l'orientation générale de cette future institution. On peut notamment imaginer que les « réserves visibles " prévues sur ses plans dans une sorte de silo transparent n'apporteront que peu d'information sur les instruments de musique qui y seront exposés.

30 - Un musée d'ethnographie, de société(s) ou de civilisation(s) : une telle institution apparaît sans doute comme l'espace le mieux adapté à la présentation de la musique dans une perspective respectant les exigences de l'ethnomusicologie. Le défi en un tel lieu réside essentiellement dans le choix de cas susceptibles de mettre en lumière quelques principes fondamentaux. Le "Salon de musique » du Musée de l'Homme demeure à cet égard un bonne exposition de référence, même si sa muséographie mériterait d'être revue. La section musicale du Musée Canadien de la Civilisation, présentée par le petit film « Géographie musicale », paraît en revanche moins convaincante, ne serait-ce qu'en raison de l'arbitraire du critère de répartition choisi. Le regroupement des instruments de musiques par pays (suivant un ordre alphabétique: Afghanistan, Albanie, Algérie, Allemagne, Angola, etc.) procède en effet d'une vision pour le moins sommaire.

31 - Un musée à vocation "nationale » : plusieurs projets de collaboration hors d'Europe avec des chercheurs locaux ont été évoqués, en particulier en Éthiopie par Olivier Tourny, au Yémen par Jean Lambert et en Bolivie par Rosalia Martínez. Cette dernière souligne notamment que la situation économique de «nos terrains» devrait, pour des raisons éthiques, nous inciter à l'économie des moyens utilisés dans l'exposition de leurs musiques. Les risques de dérives ou de raccourcis à caractère idéologique et nationaliste dans ce type d'institutions ont par ailleurs été soulignés.

32 - Des expositions thématiques : ces occasions sont sans doute celles qui permettent de présenter une musique dans les meilleures conditions. De manière générale, les lieux d'exposition doivent être «mutables » et non polyvalents, selon l'expression de Michel Colardelle, afin que leur géographie puisse mettre en évidence la construction de différences et de parentés. Joaquim Pais de Brito souligne que la musique était l'objet même de son exposition sur le fado à Lisbonne, réalisée «à partir d'une construction 
intellectuelle destinée à révéler des faits » et mettant en évidence des thèmes comme la ville, la nuit ou la ritualisation du moment musical. Elle aussi centrée sur le son, l'expérience menée par Pantxoa Etchegoin sur le chant basque divisait pour sa part l'espace d'exposition en trois zones consécutives: une zone d'immersion, une zone interactive et une zone d'écoute sélective. Quant à l'exposition sur les musiciens de rues montée au Musée des Arts et Traditions Populaires de Paris, elle a permis, selon Florence Gétreau, de mettre l'accent sur la "relation dialectique existant entre sources écrites et informations de terrain, entre histoire et ethnologie ».

\section{Que montrer de la musique?} musique? », «que dire par la musique? » ou encore « que lui faire dire? " La polysémie de tout événement musical mérite en effet d'être traitée de manière aussi adéquate que possible. Bernard Lortat-Jacob remarque que, la musique étant déjà en soi une représentation mentale («la musique est toujours plus que de la musique», selon la formule bien connue de Gilbert Rouget), la tâche essentielle du muséologue est de représenter cette représentation. Il ajoute qu'à cet égard, nous pourrons certainement soulever plus de problèmes qu'apporter de réponses.

es premiers objets à apparaître dans toute exposition de musique sont, presque inévitablement, les instruments. La présentation de ces « outils du musicien » est assortie de questions touchant à leur lutherie et à leur typologie, mais aussi à leur valeur symbolique. "L'instrument porte en lui la musique qu'il génère", affirme Philippe Bruguière, qui ajoute qu' « un public curieux d'un instrument sera forcément aussi curieux de sa musique». Une exposition organologique doit être complétée par la présentation des voix et, plus largement, du son, qui pose le problème de son immatérialité et donc des moyens à mettre en œuvre pour sa représentation. La musique comporte effectivement toujours le son, mais elle n'est pas le son; elle n'est en tout cas pas que le son, ni même que le son socialisé.

La musique en tant que pratique individuelle et collective, sa fonction et son contexte sont nécessairement au centre de toute recherche ethnomusicologique. De telles préoccupations, rappelle François Borel, étaient déjà à la base d'une exposition comme " Musique et sociétés ", montée en 1977 à Neuchâtel où, vingt ans plus tard, « Pom pom pom pom » prolongeait le propos par une réflexion sur la musique en tant que «bandeson de notre époque ", élaborée sur la base d'une série d'installations sonores.

Intentionnellement ou non, la représentation du monde proposée dans un musée est un acte politique. Il est évident que la controverse parisienne entre défenseurs du Musée de l'Homme et promoteurs de celui des « Arts et Civilisations » au quai Branly oppose deux visions de notre société, y compris dans son rapport aux autres. Mais il est à craindre que les non-dits et les atermoiements en arrivent à enterrer toute possibilité de débat serein et constructif. Autre constat: l'échec de l'«Esplanade des mondes", le projet d'un nouveau musée d'ethnographie à Genève, désavoué par la population locale suite à un référendum soutenu par le milieu du marché de l'art. L'analyse des mécanismes de cette défaite par Laurent Aubert tend à démontrer que, derrière les discours sur la culture et leurs présupposés théoriques, se cachent parfois des enjeux politiques et économiques d'un poids considérable.

Cahiers d'ethnomusicologie, 15 | 2011 


\section{Comment présenter la musique?}

37 L'image de la musique présentée dans un musée est une image recréée, qui «bouscule nécessairement la tradition", selon l'expression de Gilles Delebarre. Elle requiert la subjectivité et la créativité du muséographe-scénographe, tout en suscitant un rapport d'intersubjectivité entre producteurs (les musiciens), médiateurs (les ethnomusicologues, les muséographes) et récepteurs (les publics) de musique. Il faut tabler sur le fait qu'une exposition est en soi la transposition d'une réalité culturelle en un langage qui n'est pas le sien.

Ce langage comporte notamment des moyens picturaux, graphiques, audio-visuels et multimédia qui, en tout cas pour les derniers, n'en sont encore qu'à la phase expérimentale. L'usage du son (par haut-parleurs, sous casque ou en " douche ») doit être appliqué avec discernement afin de conjuguer écoute fine et convivialité, tout en ménageant un bon équilibre entre son qualifié et silence qualifié. De l'avis général, l'usage de casques engendre l'isolement du visiteur qui, par l'«effet zombie» qu'il suscite (François Borel), "est le contraire de l'écoute participative» vers laquelle tend la muséographie moderne (Florence Gétreau). A cet égard, les outils de sonorisation conçus et présentés par le "designer sonore " Luc Martínez offrent des solutions concrètes : on se souviendra du haut-parleur hyperfocal, projetant le son de façon aussi directionnelle qu'un spot lumineux, ou, à l'inverse, de celui permettant de distribuer le son sur une surface plane comme une vitre.

La représentation visuelle de la musique fait partie du champ de l'ethnomusicologie, y compris celle des formes et des structures qui en constituent le " corps sonore ». Le CDRom publié sur les Pygmées Aka démontre à cet égard le potentiel, tant cognitif que ludique, d'un tel produit qui, selon Susanne Fürniss, permet notamment de transmettre d'une façon relativement simple les résultats de l'analyse musicale. Marc Chemillier insiste pour sa part sur la nécessité de développer une véritable écriture multimédia afin de permettre le partage des représentations mentales de la musique. Il soulève en outre la question de savoir sur quoi faire interagir l'utilisateur.

\section{A qui s'adresse-t-on?}

Le musée en tant que lieu de vulgarisation de la connaissance a suscité des positions contrastées : Gilles Dellebarre insiste sur la nécessité de ne pas être trop pointu, alors que Bernard Lortat-Jacob affirme au contraire que nous avons affaire à un savoir souvent très complexe et qu' «à vouloir trop schématiser, on perd tout ». Ce à quoi Michel Colardelle rétorque que "le musée est un média simple et pauvre ». Quant à Rosalia Martínez, elle souligne qu'une démarche muséale est d'abord destinée à un public non averti, et qu'il est donc important de resituer la musique dans une expérience globale, d'où la nécessité de l'interdisciplinarité.

Il est clair qu'il faut viser tous les publics, sollicitant chacun selon son mode de perception et son niveau de compréhension propres. De l'avis général, une bonne muséographie doit ainsi conjuguer des approches du fait musical à caractère ludique, émotionnel, esthétique et didactique afin d'établir une dialectique avec chacun selon la nature de ses motivations. 

connaissance, c'est aussi en connaitre les outils spécifiques et faire preuve d'imagination pour y exprimer ce qui mérite d'y être dit et qui ne pourrait pas être dit ailleurs. Ainsi, en délimitant le propos, en le condensant, on peut lui donner plus de force. L'ethnomusicologue-muséographe est un maillon d'une chaîne qui comporte, en amont, les musiciens (les faiseurs de musique) et, en aval, les scénographes, les techniciens et les artisans du musée (les faiseurs d'expositions). Le rôle de l'ethnomusicologue de musée est celui d'un traducteur, d'un passeur de musiques grâce auquel, à travers une perception différée du fait musical, les visiteurs peuvent découvrir quelque chose sur les autres, et donc sur eux-mêmes.

Laurent Aubert 
